\title{
Evaluation of Potential Hepatotoxicity Induced by Bortezomib
}

\author{
Duygu Mutluay', Yanad Abou Monsef² \& Gözde Yücel Tenekeci²
}

\begin{abstract}
Background: Bortezomib, an inhibitor of $26 \mathrm{~S}$ proteasome, is an anti-cancer therapeutic agent used in different cancer types. It leads to the arrest of the cancerous cell cycle by inhibiting angiogenesis and inducing apoptosis. Liver is the vital organ for detoxification and excretion of toxic products. The treatment with chemotherapy is a challenge, drugs are used to destroy cancer cells, but healthy cells can be affected during cancer treatment as well. The main objective of this study was to analyze the histopathological and biochemical effects of bortezomib on liver.

Materials, Methods \& Results: Twenty-four female C57BL/6 mice were distributed into 4 groups, bortezomib injected treatment groups (Btz1, Btz2) and saline injected control groups $(\mathrm{C} 1, \mathrm{C} 2)$. Bortezomib and saline were treated twice per week for 6 weeks and sacrificed at the end of one day (Btz1, C1) and 4 weeks (Btz2, C2) after the last injection. Liver samples were examined for histopathological analysis and the serum samples processed for biochemical analysis. Tissue samples were fixed, routinely processed, sectioned, and stained with Hematoxylin and Eosin (H\&E). Periodic Acid-Schiff (PAS), Sudan Black staining and Masson's trichrome histochemical staining methods were performed to characterize the lesions. Histopathological analysis of the Btz1 and Btz2 groups revealed acute hepatic morphological changes such as hepatocellular swelling (cloudy swelling), necro-inflammatory reaction, and increased mononuclear polyploidy. Based on the negative staining with PAS and Sudan Black staining, hepatocellular swelling was diagnosed as hydropic degeneration. Necro-inflammatory reaction observed in the form of acute hepatitis was composed of mainly mononuclear cell infiltration accompanied by multifocal necrotic foci. Kupffer cell proliferation was observed in parallel with degenerative and necrotic changes. An Increase in hepatocellular mononuclear polyploidy visualized as hepatocytes with a single enlarged nucleus was detected in all liver sections of Btz1 and Btz2 groups. Individual cases of cholestasis $(n=1)$ and mild hepatic fibrosis $(n=1)$ were also reported. Significant elevated levels of alanine aminotransferase (ALT), aspartate transaminase (AST), alkaline phosphatase (ALP) and gamma-glutamyl transferase (GGT) were detected in bortezomib treated groups.

Discussion: Few clinical cases reported liver injury related to bortezomib used for cancer treatment. However, the liver was not considered as a target for bortezomib treatment. Our data suggesting that bortezomib caused liver damage and induces elevations in serum levels. The reported hepatic lesions including hepatocellular swelling, acute hepatitis and mononuclear polyploidy were mainly mild and moderate in severity. The increase of polyploidy in liver tissue of mice treated with bortezomib in this study was explained as a reaction of the liver facing the drug-induced hepatic damage. The mechanism leading to the hepatotoxicity of bortezomib treatment is not known but the production of a toxic metabolite through its metabolism in the liver can be suggested. Moreover, no recovery was also observed in histopathological and biochemical analyses suggesting that the bortezomib effect is non-reversible four weeks after the drug was withdrawn. Patients should be informed about the possibility of acute drug-induced hepatitis and hepatotoxicity of this chemotherapeutic agent after the treatment.
\end{abstract}

Keywords: bortezomib, cancer, chemotherapy, hepatotoxicity, liver, proteasome inhibitor. 


\section{INTRODUCTION}

Bortezomib is the first in class proteasome inhibitor used as an anti-cancer drug which is known as a proteasome inhibitor. The proteasome is an essential element of the ubiquitin-proteasome system (UPS), responsible for regulation and degradation of the majority of eukaryotic intracellular protein turnover [5]. Bortezomib acts as a chemotherapeutic agent by leading cancerous cells to cell cycle arrest, inhibiting angiogenesis and inducing apoptosis [12,19,31]. Bortezomib was mainly used the treatment of cancers such as multiple myeloma, lymphoma, ovarian cancer, lung cancer, prostate cancer, pancreatic cancer, melanoma, glioblastoma [3] and various disease like congenital erythropoietic porphyria [2] and graft-versus host disease [16]. Bortezomib can also be used in veterinary field for treating canine osteosarcoma [20] which sarcomas represent $15 \%$ of cancers in dogs [24] and canine malignant melanomas [10].

Chemotherapeutic agents can treat various cancer types although they can cause serious side effects in the body [7]. Animal studies showed effects of bortezomib on the liver; increases liver weight due to hepatocellular hypertrophy, vacuolization and pigment deposition in hepatocytes [27]. Studies also suggest that bortezomib elevates the liver enzyme levels and can be the cause of liver injury in patients treated for multiple myeloma with bortezomib [14,27].

Liver is one of the first organ exposed to drugs and chemotherapy, however there is very limited information about effects of bortezomib on histopathology of liver and liver cells. In this study we aimed to the investigate the potential side effects of bortezomib on liver.

\section{MATERIALS AND METHODS}

Animals

Twenty-four adult C57BL/6 female mice (aged 9 weeks) were used in the study. The mice were placed in a vivarium with controlled temperature and humidity (12-h light: dark cycle) and were fed ad libitum. General condition and food intake were monitored daily and body weights of mice were measured twice weekly before the injections.

\section{Drug preparation and treatment}

Bortezomib $^{1}(3.5 \mathrm{mg})$ was purchased from the pharmacy and suspended in $3.5 \mathrm{~mL}$ sterile $(0.9 \%)$ saline solution. Injections were administered by in- traperitoneally in a volume of $0.01 \mathrm{~mL} / \mathrm{g}$ body weight via 28 -gauge needles. Animals were divided into 4 different groups containing 6 animals in each group ( $n=6 /$ group) as follows: Btz treatment groups 1 and 2 (Btz1, Btz2) and control groups 1 and 2 (C1, C2). Btz groups injected $1 \mathrm{mg} / \mathrm{kg}$ Btz intraperitoneally (i.p.) twice per week for 6 weeks, on days 1-4-8-1115-18-22-25-29-32-36-39 while control groups were given $1 \mathrm{mg} / \mathrm{kg} 0.9 \%$ saline i.p. as a vehicle solution on the same days as treatment groups [4]. Btz dose was chosen to be $1 \mathrm{mg} / \mathrm{kg}, 2$ times a week $(42.8 \mathrm{mg} / \mathrm{m} 2)$, similar to the standard dose used cancer patients with a schedule of $1.3 \mathrm{mg} / \mathrm{m}^{2} 4$ times each, 21 days during 8 cycles $\left(41.6 \mathrm{mg} / \mathrm{m}^{2}\right)[4,25,30]$. In this study, we administered 3 cycles of treatment consecutively. The $1 \mathrm{st}$ cycle of therapy was constituted by manufacturer on days 1, 4, 8 and 11 . The mice from Btz1 and $\mathrm{C} 1$ group were sacrificed at 40th day post-injection. Afterwards, mice from Btz2 and C2 group were left untreated for a recovery period of 4 weeks before euthanized to assess the potential recovery in liver tissue [4].

\section{Biochemical analysis}

Blood was collected from control and Btz treatment groups by cardiac puncturing from the heart and was allowed to clot by leaving it at room temperature then the serum was separated by centrifugation at $662 \mathrm{~g}$ for $10 \mathrm{~min}$ in a refrigerated centrifuge. The serum was used to measurement of ALT (alanine aminotransferase), AST (aspartate transaminase), ALP (alkaline phosphatase) and GGT (Gamma-glutamyl Transferase). The samples were analyzed by using automatic biochemistry analyzer (Randox Brand RX Daytona Plus)².

\section{Histopathological examination}

Liver specimens from different groups were isolated and fixed in $10 \%$ neutral-buffered formaldehyde solution. After dehydration in a growing series of ethanol, the samples were blocked in paraffin, and sectioned $(4 \mu \mathrm{m})$ by a microtome. Sections were automatically stained with Hematoxylin and Eosin $(\mathrm{H} \& \mathrm{E})^{3}$ and were mounted with glass coverslips. The slides were then evaluated under the light microscope (Olympus BX51) by 2 experienced investigators unaware of the experimental groups and the lesions semi-quantitively assessed in 4 different histological areas of the liver (portal, periportal, parenchymal and septal). The detected hepatic lesions were graded from 0 (normal) to 3 (severe lesions). Hepatocellular vacu- 
olation and necrosis were scored based on the percentage of the area affected, into the following categories: $-(<5 \%),+(5-33 \%),++(34-66 \%)$ and $+++(>66 \%)$ at $100 \times$ magnification. As for inflammation, 5 different fields of $100 \times$ magnification were evaluated and the number of inflammatory foci per field was counted and the average was scored into: - normal $(<0.5$ foci $),+$ slight (0.5-1.0 foci), ++ moderate (1.0-2.0 foci) and +++ severe ( $>2.0$ foci) [17].

\section{Histochemistry}

Special histochemical stains such as Periodic Acid-Schiff (PAS) ${ }^{3}$, Sudan Black staining and Masson's trichrome ${ }^{3}$ staining methods were also performed to further characterize the lesions. Periodic Acid-Schiff (PAS) and Sudan Black staining were used respectively to detect hepatocellular polysaccharide and lipid components while Masson's trichrome staining was performed to evaluate hepatic fibrosis. PAS, Sudan Black and Masson's trichrome staining were done according to the appropriate staining procedures [20].

\section{Statistical analysis}

Mean \pm standard error (SEM) was calculated by using SPSS/PC computer program (version 22.0, SPSS Inc). Means were tested by using one-way analysis of variance (ANOVA), followed by Duncan post hoc test to evaluate the significant differences between treatment and control groups. The values of $P<0.001$ were considered statistically significant.

\section{RESULTS}

\section{Histopathological effects of bortezomib on liver}

The liver sections of control groups showed normal histological architecture of the lobules and normal structure of the hepatocytes arranged in radiating cordons. Small infiltrates of lymphocytes (less than 5 cells per foci) were observed in few control sections. Hepatocytes with a large nucleus (Polyploid hepatocytes) and binucleated hepatocytes were frequently observed.

Btz1 and Btz2 groups exhibited various degrees of hepatic histopathological changes compared to $\mathrm{C} 1$ and $\mathrm{C} 2$ groups (Table 1). Hepatic alterations were the following: hepatocellular vacuolation, inflammatory infiltrates, hepatic necrosis, and increased polyploidy. The lobular architecture was preserved in the majority of Btz1 and Btz2 mice. However, diffuse vacuolar hepatopathy was observed in Btz1 and Btz2 liver samples. It can be described as a degenerative change exhibited by swelling of the hepatocytes with pale staining cytoplasm and intracytoplasmic vacuolar structures. Based on the histopathological features of vacuolar hepatopathy and the negative staining with PAS and Sudan Black staining, these vacuolar changes were diagnosed as hepatocellular swelling (cloudy swelling, hydropic degeneration) [Figure 3]. Proliferation of the Kupffer cells known as the star shaped liver macrophages was identified in the degenerative areas. While the intensity of hepatocellular swelling varied from mild to moderate in Btz1 group, it ranged from moderate to severe in Btz2 group which indicates an increased hepatocellular damage in this group (Figure 1).

Mild or moderate inflammatory reaction composed of mononuclear cell infiltration (mainly lymphocytes) was observed in the portal tracts and/or in the parenchymal lobes (intralobular) of Btz1 and Btz2 groups (Figures 1 and 2). Inflammatory infiltrates were in the form of clusters of more than 5 inflammatory cells. Multifocal hepatocellular necrotic areas associated with the intralobular mononuclear cell infiltration and Kupffer cell proliferation were noticed. As a result, based on the previously described necro-inflammatory reaction, the majority of Btz1 and Btz2 were diagnosed with acute hepatitis. Dilatation and active hyperemia of the portal vessels and sinusoids was observed in parallel to the necro-inflammatory reaction. The severity of the inflammatory infiltration was almost unchanged between Btz1 and Btz2 groups.

Mild fibrosis showing thickening of the portal tracts due to the growth of connective tissue strands was only observed in one case of Btz1 group. On the other hand, another individual case of Btz1 group revealed a mild diffuse intracytoplasmic accumulation of yellow bile granules in the hepatocytes and was diagnosed as cholestasis. The number of polyploid hepatocytes or mononuclear polyploidy known as hepatocytes with a single enlarged nucleus compared to the nuclei of normal hepatocytes was increased in Btz1 and Btz2 groups compared to $\mathrm{C} 1$ and $\mathrm{C} 2$ groups. This feature was detected in almost all liver sections of Btz1 and Btz2 groups (Figure 2).

\section{Effects of Bortezomib on biochemical parameters}

Serum enzymes such as AST, ALT, ALP and GGT levels were elevated for control and treatment groups to assess the liver injury. ALT, AST, ALP and 
GGT have been widely used as a hepatocellular injury markers. AST, ALT enzymes mainly were found in liver and muscle while ALP were found in the liver, biliary cells and kidneys [9]. GGT were located in the hepatocytes, and biliary ducts of the liver which ALP and GGT are more specific for hepatobiliary changes [8].
ALT, AST, ALP and GGT levels were significantly increased in the Btz1 and Btz2 groups compared to $\mathrm{C} 1$ and C2 groups $(P<0.001)$ [Table 2]. When ALT level was compared between Btz1 and Btz2 groups, we detected that ALT level was significantly elevated in Btz2 group after 4 weeks recovery period $(P<0.001)$ [Table 2].

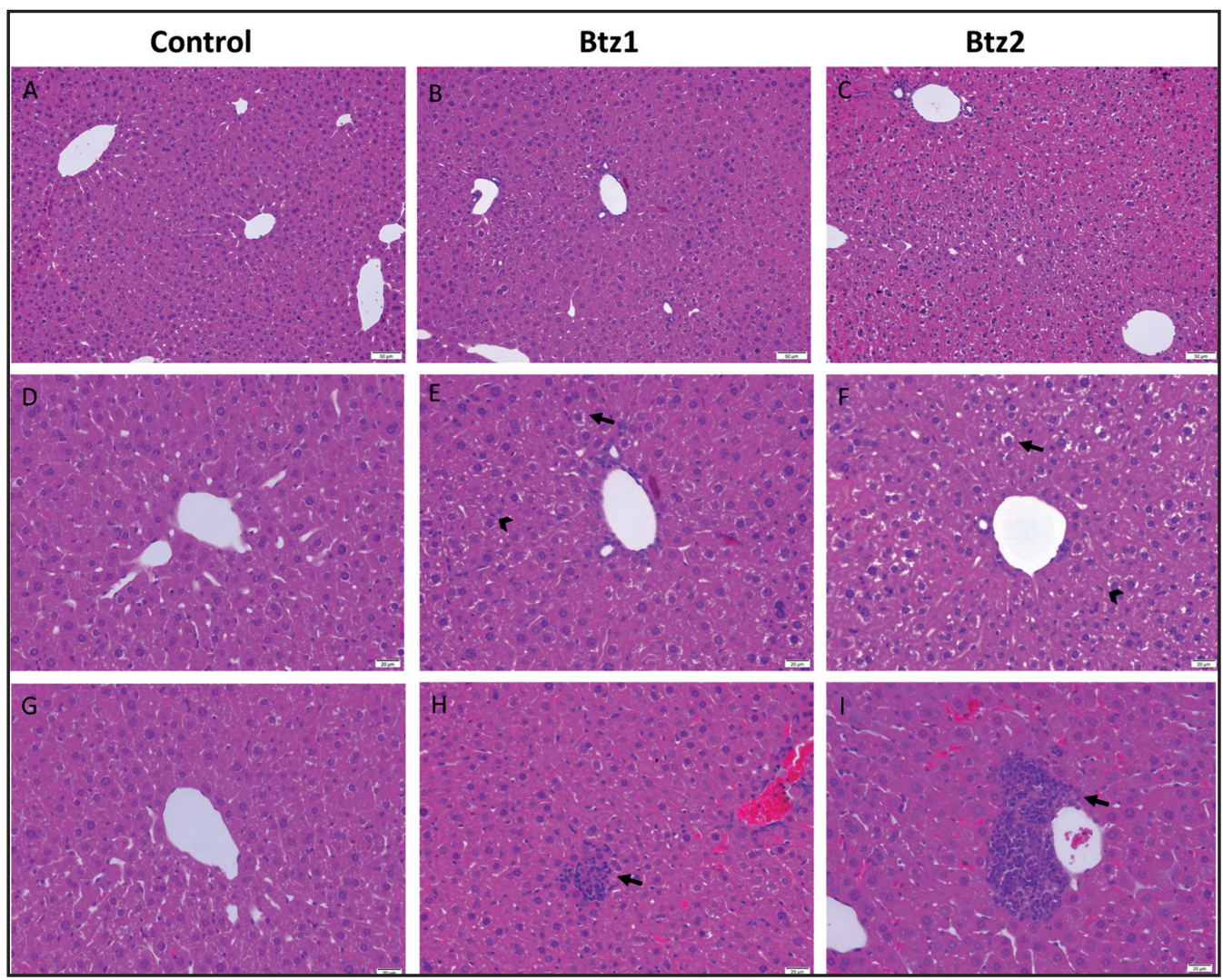

Figure 1. Histopathological features of liver sections from Control (A, D \& G) and Experimental groups (Btz1 and Btz2) [H\&E]. A, D \& G- Cross sections of healthy liver samples. B, C, E \& F- Hepatocellular swelling (arrow) and activated Kupffer cells (arrowhead). H \& I- Parenchymal inflammatory infiltrates (arrow) accompanied by necrosis of the underlying parenchymal cells.

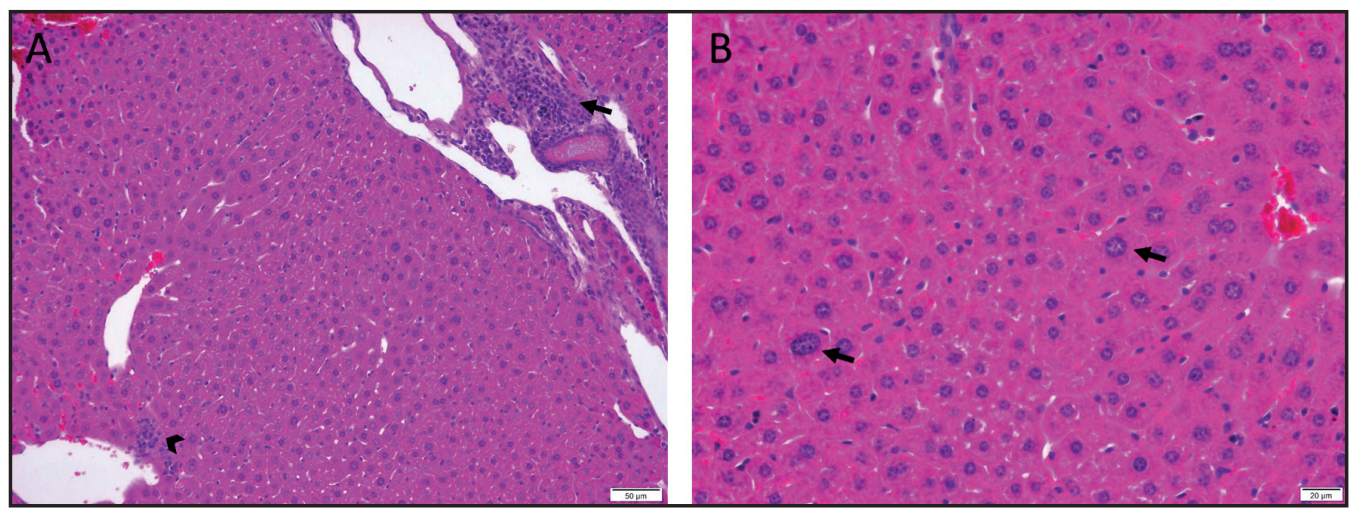

Figure 2. Acute hepatitis and polyploidy in Btz1 groups. A- Centrilobular (arrowhead) and portal inflammatory clusters [H\&E; 200x]. B- Mononuclear polyploidy: enlarged hepatocyte nuclei (arrow) [H\&E; 400x]. 


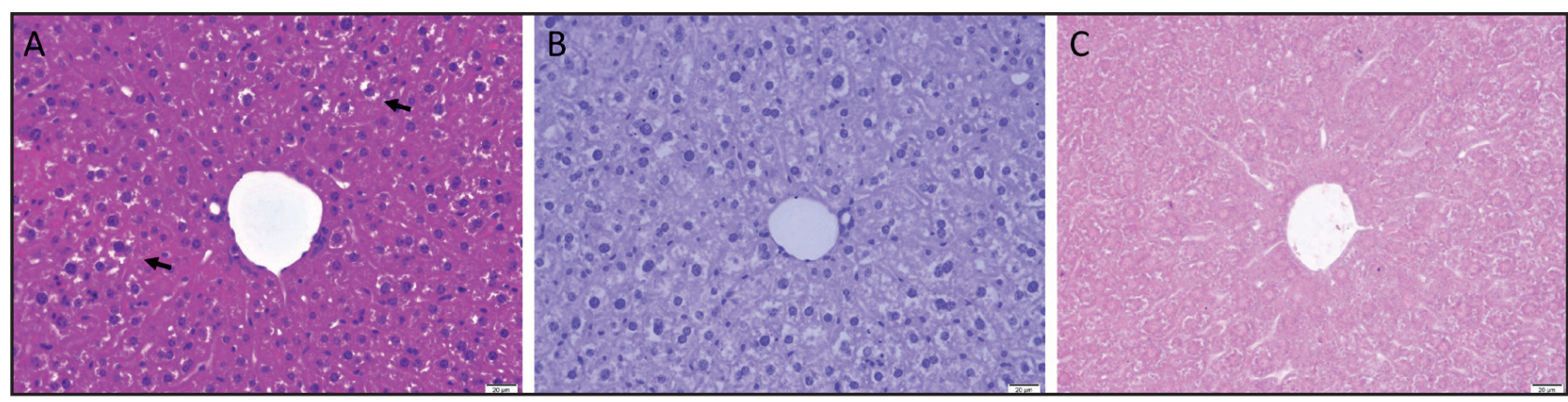

Figure 3. Vacuolar hepatopathy in Btz1 group (A, arrows) showing negative staining with PAS (B) and Sudan black (C) staining methods.

Table 1. Semiquantitative scoring of hepatic histopathologic features in control $(\mathrm{C} 1, \mathrm{C} 2)$ and experimental groups (Btz1, Btz2).

\begin{tabular}{ccccc}
\hline Histopathologic features & C1 & C2 & Btz1 & Btz2 \\
\hline Hepatocellular vacuolation & - & - & + & ++ \\
Hepatocellular necrosis & - & - & + & ++ \\
Inflammation & - & - & + \\
\hline
\end{tabular}

+: mild; ++: moderate; +++: strong.

Table 2. Biochemical parameters (mean $\pm \mathrm{SEM})$ in mice sacrificed, 1 day $(\mathrm{C} 1, \mathrm{Btz} 1)$ and 4 weeks $(\mathrm{C} 2$, Btz2) after the last bortezomib or saline injection $(1 \mathrm{mg} / \mathrm{kg})$.

\begin{tabular}{ccccc}
\hline Group & ALT $(\mathrm{U} / \mathrm{L})$ & AST $(\mathrm{U} / \mathrm{L})$ & ALP $(\mathrm{U} / \mathrm{L})$ & GGT (U/L) \\
\hline C1 & $53.50 \pm 4.28^{\mathrm{a}}$ & $117.66 \pm 16.99^{\mathrm{a}}$ & $68.00 \pm 2.26^{\mathrm{a}}$ & $6.50 \pm 0.56^{\mathrm{a}}$ \\
Btz1 & $100.50 \pm .80^{\mathrm{b}}$ & $323.50 \pm 11.98^{\mathrm{b}}$ & $150.33 \pm 8.77^{\mathrm{b}}$ & $12.50 \pm 0.61^{\mathrm{b}}$ \\
C2 & $51.50 \pm 3.82^{\mathrm{a}}$ & $118.83 \pm 14.55^{\mathrm{a}}$ & $72.00 \pm 3.18^{\mathrm{a}}$ & $7.00 \pm 0.36^{\mathrm{a}}$ \\
Btz2 & $125.50 \pm 4.15^{\mathrm{c}}$ & $344.66 \pm 12.70^{\mathrm{b}}$ & $156.50 \pm 7.96^{\mathrm{b}}$ & $12.66 \pm 0.49^{\mathrm{b}}$ \\
\hline
\end{tabular}

$P$

${ }^{\text {a-c } V a l u e s ~ i n ~ t h e ~ s a m e ~ c o l u m n ~ w i t h ~ d i f f e r e n t ~ s u p e r s c r i p t s ~ a r e ~ s t a t i s t i c a l l y ~ d i f f e r e n t ~}\left({ }^{*} P<0.001\right)$. Control groups 1 and $2(\mathrm{C} 1, \mathrm{C} 2)$. Bortezomib treatment groups 1 and 2 (Btz1, Btz2).

\section{DISCUSSION}

Previous studies indicated that enhanced activation of proteasome pathway underlies the pathogenesis of many diseases in human and animals [1]. Studies reported that bortezomib can be safely used to treat the several diseases in dogs $[1,21,32]$. Especially bortezomib is effective on canine malignant melanomas known as too aggressive and fatal neoplasms [10] and osteosarcoma that is the fourth most common malignancy in pure-breed dogs $[6,22]$. Bortezomib also can be used the treatment of Golden retriever muscular dystrophy (GRMD) by healing the appearance of GRMD muscle fibers and reducing the infiltration of inflammatory cells [1]. Moreover, bortezomib can treat equine endotoxemia which is a serious disease with a high mortality and endotoxin associated laminitis [28]. All those studies showed that bortezomib is well tolerated in some animals and might have a potential as a therapeutic agent to treat several diseases.

The liver is a central organ, responsible for metabolic detoxification and excretion of toxic products [23]. Unfortunately, various chemotherapeutic agents can function nonspecifically and damage both malignant and healthy tissues [26]. Bortezomib has been reported to cause liver injury in a few clinical cases during cancer treatment [14,27]. Bortezomib related hepatic lesions such as drug-induced hepatitis, cholestasis and hepatocellular vacuolization have been indicated but the liver was not considered as a target 
for this drug's toxicity [13,14,27]. In accordance with previous studies, bortezomib induced changes included hepatocellular swelling, acute hepatitis and increase in the mononuclear polyploidy in our present study. Grading of the reported hepatic lesions showed a mild to moderate severity and no chronic hepatic changes were reported except for 1 individual case belonging to the Btz1 group presenting a mild fibrosis. These previously cited features reported in both experimental groups (Btz1 and Btz2 groups) suggest the reversibility of the drug induced hepatic lesions and its possibility to be treated in clinical practice.

Jain et al. [13] reported a cholestatic pattern of injury related to Bortezomib treatment in a 54-year-old non-alcoholic man diagnosed with multiple myeloma. Identical type of hepatic injury or biliary alteration was not reported in our study except for 1 individual case from Btz1 group showing bile pigment accumulation in hepatocytes cytoplasm. These mild cholestasis features are thought to be secondary to drug-induced hepatic injury in the experimental and thus considered as an intrahepatic cholestasis.

Increase in polyploidy of hepatocytes was remarkable in Btz1 and Btz2 when compared to liver samples from control groups. Polyploidy is a feature of liver growth only exhibited in some mammal's tissues such as the liver, heart and skeletal muscle. Binucleated hepatocytes turn into polypoid hepatocytes through the failure of karyokinesis [15]. According to Kreutz et al. [15] a large proportion of the hepatocytes in mice liver $(75 \%)$ are polyploid. In a study by Toyoda et al. [29], related to the changes of hepatocytes polyploidy during human chronic viral hepatitis, the percentage of mononuclear hepatocytes increased in patients with high hepatitis activity and marked fibrosis. This may explain the increase of polyploidy in liver tissue of mice that developed acute hepatitis and degenerative changes related to the treatment with bortezomib in this study. Moreover, the liver can recover after toxicant withdrawal, in our study we did not observe any recovery on the contrary, hepatocellular vacuolation and necrosis were stronger in Btz2 group compared to $\mathrm{C} 1, \mathrm{C} 2$ and Btz1groups.

In the present study we assessed the enzyme biomarkers such as AST, ALT, ALP and GGT to evaluate the liver structural integrity and damage. We specifically analyzed more than one liver enzyme that have been used as a marker of hepatoxicity because there is no specific enzyme for liver damage. When there is an injury in the liver, enzymes are increased in the liver and spilled into the blood system [9]. We detected that AST, ALT, ALP and GGT enzyme levels were significantly elevated after bortezomib administration. Based on serum levels, we also determined that bortezomib effect is non-reversible neither 1 day or 4 weeks after the drug was withdrawn. In Btz2 group, unlike other enzyme levels ALT which is the most liver specific of the liver enzymes was also significantly increased compared to $\mathrm{C} 1, \mathrm{C} 2$ and Btz1 groups. Compatible with our biochemistry findings, severe increase in AST, ALT and LDH levels was associated with bortezomib in 2 case reports [14,27]. No recovery was also observed in serum levels compatible with our histopathology findings. According to our results we suggest that bortezomib therapy is associated with increase in liver enzyme levels.

Our study indicated that hepatocellular injury and elevated serum enzyme levels were induced after bortezomib treatment however the mechanism of this drug is not known. In vitro studies on human liver microsomes and human complementary DNA-expressed cytochrome P450 (CYP) isoenzymes indicated that several isoenzymes such as CYP3A4, CYP2C19, and CYP1A2 mediated bortezomib metabolism [11]. Bortezomib is metabolized in the liver primarily by cytochrome CYP3A4 pathway and liver injury may be related to production of a toxic intermediate [18].

\section{CONCLUSIONS}

In accordance with the alteration in histopathological and biochemical results, we suggest that bortezomib treatment causes liver injury and damage and induce elevations in serum levels. All these findings showed the toxic effects of bortezomib on the liver. We are suggesting that patients should be taken into account the possibility of acute drug-induced hepatitis and drug may cause hepatotoxicity after treatment. Our findings may help the clinicians to plan, address and inform the patients who are being treated with bortezomib about the potential negative effects of this drug.

\footnotetext{
MANUFACTURERS

${ }^{1}$ Millennium Pharmaceuticals. Cambridge, MA, USA.

${ }^{2}$ Randox Laboratories Ltd. Crumlin, UK.

${ }^{3}$ Merck KGaA. Darmstadt, Germany.

${ }^{4}$ Olympus Corporation. Shinjuku-ku City, Tokyo, Japan.
} 
Acknowledgments. The authors would like to thank MSc. Cennet Sinem Coban who helped with animal work, tissue collection and Dr. Volkan Ipek for his help with tissue collection and processing.

Ethical approval. Animal care was performed in accordance with the animal ethics principles of Burdur Mehmet Akif Ersoy
University, and approval of the experimental protocol obtained by the Veterinary Faculty Experimentation Ethics Committee of Mehmet Akif Ersoy University (approval ID 348-2017).

Declaration of interest. The authors report no conflicts of interest. The authors alone are responsible for the content and writing of paper.

\section{REFERENCES}

1 Araujo K.P., Bonuccelli G., Duarte C. N., Gaiad T.P., Moreira D.F., Feder D., Belizario J.E., Miglino M.A. Lisanti M.P. \& Ambrosio C.E. 2013. Bortezomib (PS-341) treatment decreases inflammation and partially rescues the expression of the dystrophin-glycoprotein complex in GRMD dogs. PloS One. 8(4): e61367.

2 Blouin J.M., Duchartre Y., Costet P., Lalanne M., Ged C., Lain A., Millet O., de Verneuil H. \& Richard E. 2013. Therapeutic potential of proteasome inhibitors in congenital erythropoietic porphyria. Proceedings of the National Academy of Sciences (Proceedings of the National Academy of Sciences of the United States of America). 110: 1823818243.

3 Brignole, C., Marimpietri D., Pastorino F, Nico B., Di Paolo D., Cioni M., Piccardi F, Cilli M., Pezzolo A., Corrias M.V., Pistoia V., Ribatti D., Pagnan G. \& Ponzoni M. 2006. Effect of bortezomib on human neuroblastoma cell growth, apoptosis, and angiogenesis. Journal of the National Cancer Institute. 98(16): 1142-1157.

4 Bruna J., Udina E., Alé A., Vilches J.J., Vynckier A., Monbaliu J., Silverman L. \& Navarro X. 2010. Neurophysiological, histological and immunohistochemical characterization of bortezomib-induced neuropathy in mice. Experimental Neurology. 223(2): 599-608.

5 Dick L.R. \& Fleming P.E. 2010. Building on bortezomib: second-generation proteasome inhibitors as anti-cancer therapy. Drug Discovery Today. 15(5-6): 243-249.

6 Dobson J.M. 2013. Breed-predispositions to cancer in pedigree dogs. International Scholarly Research Network Veterinary Science. 2013(11): DOI 10.1155/2013/941275

7 El-Sayyad H.I., Ismail M.F., Shalaby F.M., Abou-El-Magd R.F., Gaur R.L., Fernando A., Raj M.H. \& Ouhtit A. 2009. Histopathological effects of cisplatin, doxorubicin and 5-flurouracil (5-FU) on the liver of male albino rats. International Journal of Biological Sciences. 28(5): 466-473.

8 Everds N.E. 2015. Evaluation of clinical pathology data: correlating changes with other study data. Toxicologic pathology. 43(1): 90-97.

9 Hasan K.M.M., Tamanna N. \& Haque M.A. 2018. Biochemical and histopathological profiling of Wistar rat treated with Brassica napus as a supplementary feed. Food Science and Human Wellness. 7(1): 77-82.

10 Ito K., Kobayashi M., Kuroki S., Sasaki Y., Iwata T., Mori K., Kuroki T., Ozawa Y., Tetsuka M., Nakagawa T., Hiroi T., Yamamoto H., Ono K., Washizu T. \& Bonkobara M. 2013. The proteasome inhibitor bortezomib inhibits the growth of canine malignant melanoma cells in vitro and in vivo. The Veterinary Journal. 198(3): 577-82.

11 Iwamoto T., Ishibashi M., Fujieda A., Masuya M., Katayama N. \& Okuda M. 2010. Drug interaction between itraconazole and bortezomib: exacerbation of peripheral neuropathy and thrombocytopenia induced by bortezomib. Pharmacotherapy. 30(7): 661-665.

12 Jackson G., Einsele H., Moreau P. \& Miguel J.S. 2005. Bortezomib, a novel proteasome inhibitor, in the treatment of hematologic malignancies. Cancer Treatment Reviews. 31: 591-602.

13 Jain A., Malhotra P., Suri V., Varma S., Das A. \& Mitra S. 2016. Cholestasis in a Patient of Multiple Myeloma: A Rare Occurrence of Bortezomib Induced Liver Injury. Indian Journal of Hematology and Blood Transfusion. 32(1): 181-183.

14 Kim Y., Kim K.Y., Lee S.H., Chung Y.Y., Yahng S.A., Lee S.E., Park G. \& Min C.K. 2012. A Case of Drug-Induced Hepatitis due to Bortezomib in Multiple Myeloma. Immune Network. 12(3): 126-128.

15 Kreutz C., MacNelly S., Follo M., Wäldin A., Binninger-Lacour P., Timmer J. \& Bartolomé-Rodríguez M.M. 2017. Hepatocyte ploidy is a diversity factor for liver homeostasis. Frontiers in physiology. 8: 862.

16 Li Z., Wu Q., Yan Z., Li D., Lu G., Mou W., Wu S., Pan X., Lu Q. \& Xu K. 2013. The protection and therapy effects of bortezomib in murine acute graft-versus-host disease. Transplantation Proceedings. 45: 2527-2535. 
17 Liang W., Menke A.L., Driessen A., Koek G.H., Lindeman J.H., Stoop R., Havekes L.M., Kleemann R. \& Van den Hoek A.M. 2014. Establishment of a general NAFLD scoring system for rodent models and comparison to human liver pathology. PloS one. 9(12): e115922.

18 LiverTox. 2012. Clinical and Research Information on Drug-Induced Liver Injury [Internet]. Bethesda (MD): National Institute of Diabetes and Digestive and Kidney Diseases - Bortezomib. [Updated 2017 Sep 30]. Available at https:// www.ncbi.nlm.nih.gov/books/

19 Ludwig H., Khayat D., Giaccone G. \& Facon T. 2005. Proteasome inhibition and its clinical prospects in the treatment of hematologic and solid malignancies. Cancer. 104: 1794-1807.

20 Luna L.G. 1968. Manual of Histological Staining Methods of the Armed Forces Institute of Pathology. 3rd edn. New York: McGraw Hill Book Company,186p.

21 Monahan P.E., Lothrop C.D., Sun J., Hirsch M.L., Kafri T., Kantor B., Sarkar R., Tillson D.M., Elia J.R. \& Samulski R.J. 2010. Proteasome inhibitors enhance gene delivery by AAV virus vectors expressing large genomes in hemophilia mouse and dog models: a strategy for broad clinical application. Molecular therapy: the journal of the American Society of Gene Therapy. 18(11): 1907-1916.

22 Patatsos K., Shekhar T.M. \& Hawkins C.J. 2018. Pre-clinical evaluation of proteasome inhibitors for canine and human osteosarcoma. Veterinary and Comparative Oncology. 16(4): 544-553.

23 Ramadori G. \& Cameron S. 2010. Effects of systemic chemotherapy on the liver. Annals of Hepatology. 9(2): 133143.

24 Rao S.R., Somarelli J.A., Altunel E., Selmic L.E., Byrum M., Sheth M.U., Cheng S., Ware K.E., Kim S.Y., Prinz J.A., Devos N., Corcoran D.L., Moseley A., Soderblom E., Hsu S.D. \& Eward W.C. 2020. From the Clinic to the Bench and Back Again in One Dog Year: How a Cross-Species Pipeline to Identify New Treatments for Sarcoma Illuminates the Path Forward in Precision Medicine. Frontiers in Oncology. 10: 117.

25 Reagan-Shaw S., Nihal M. \& Ahmad N. 2008. Dose translation from animal to human studies revisited. Federation of American Societies for Experimental Journal. 22(3): 659-661.

26 Rochette L., Guenancia C., Gudjoncik A., Hachet O., Zeller M., Cottin Y. \& Vergely C. 2015. Anthracyclines/ trastuzumab: new aspects of cardiotoxicity and molecular mechanisms. Trends in Pharmacological Sciences. 36: 326348.

27 Rosiñol L., Montoto S., Cibeira M.T. \& Bladé J. 2005. Bortezomib-induced severe hepatitis in multiple myeloma: a case report. Archives of Internal Medicine. 165(4): 464-465.

28 Sato H., Matsuda K., Amagai Y., Tanaka A. \& Matsuda H. 2018. Suppressive Effect of Bortezomib on LPS-Induced Inflammatory Responses in Horses. Journal of Equine Veterinary Science. 61: 114-120.

29 Toyoda H., Bregerie O., Vallet A., Nalpas B., Pivert G., Brechot C. \& Desdouets C. 2005. Changes to hepatocyte ploidy and binuclearity profiles during human chronic viral hepatitis. Gut. 54(2): 297-302.

30 Trevisan G., Materazzi S., Fusi C., Altomare A., Aldini G., Lodovici M., Patacchini R., Geppetti P. \& Nassini R. 2013. Novel therapeutic strategy to prevent chemotherapy-induced persistent sensory neuropathy by TRPA1 blockade. Cancer Research. 73(10): 3120-3131.

31 Voorhees P.M., Dees E.C., O’Neil B. \& Orlowski R.Z. 2003. The proteasome as a target for cancer therapy. Clinical Cancer Research. 9: 6316-6325.

32 Yu X., Huang S., Patterson E., Garrett M.W., Kaufman K.M., Metcalf J.P., Zhu M., Dunn S.T. \& Kem D.C. 2005. Proteasome degradation of GRK2 during ischemia and ventricular tachyarrhythmias in a canine model of myocardial infarction. American journal of physiology. Heart and Circulatory Physiology. 289(5): H1960-H1967. 